\title{
Effect of pancreas-after-kidney transplant on renal graft survival
}

Pancreas-after-kidney transplantation (PAKT) is associated with improved longterm renal allograft survival in patients with diabetes, according to a recent study.

Pancreas transplantation is known to improve survival of patients with type 1 diabetes and end-stage renal disease. Simultaneous pancreas and kidney transplantation has historically been associated with superior survival to PAKT, but improvements in immunosuppressive agents and surgical techniques have led to improved survival rates with PAKT and one study has suggested that PAKT outcomes are now superior. An advantage of PAKT is that it means that patients can receive a renal transplant from a living donor, thereby avoiding or reducing exposure to dialysis. As evidence regarding the effect of PAKT on renal allograft survival is conflicting, Browne et al. set out to determine the effect of PAKT on kidney allograft outcomes.

The researchers used data from the United States Renal Data System database from 2,776 adults who received a kidney transplant between January 1988 and August 2007, followed by a PAKT during the same time period. Data from
13,635 young adults (aged $18-40$ years) who had diabetes and had received kidneyonly transplants during the same period were used as a comparator group. Browne et al. accounted for selection bias related to the fact that not all kidney-only transplant recipients would have been eligible for PAKT by determining a propensity score for PAKT in this group, and adjusting for this score in multivariate analyses.

\section{Efforts to decrease mortality in the period after pancreas transplantation should be prioritized 77}

Early after pancreas transplantation, particularly in the first 2 weeks, the risk of kidney allograft failure was higher in PAKT recipients than in patients with kidney-only transplants. This risk equalized at 143 days after pancreas transplantation, and in the long term, multivariate analyses adjusted for propensity score showed that the risk of renal allograft failure was lower in PAKT recipients than in patients with diabetes who received kidney-only transplants (hazard ratio $0.89, P=0.05$ ). PAKT was associated with improved survival in patients with glomerular filtration rates (GFRs) of $40-49 \mathrm{ml} / \mathrm{min} / 1.73 \mathrm{~m}^{2}$, $50-59 \mathrm{ml} / \mathrm{min} / 1.73 \mathrm{~m}^{2}$, and $\geq 60 \mathrm{ml} /$ $\min / 1.73 \mathrm{~m}^{2}$, but not in patients with a GFR of $30-39 \mathrm{ml} / \mathrm{min} / 1.73 \mathrm{~m}^{2}$ (although the authors note that 10 -year allograft survival was $69 \%$ even in this group).

The researchers also found that decreased GFR at the time of pancreas transplantation was associated with an increased risk of allograft failure, although this association was not seen until 3 years after pancreas transplantation.

"In conclusion, despite an increased risk of death in the perioperative period following pancreas transplantation, PAK $[\mathrm{T}]$ was associated with a reduced risk of long-term kidney allograft failure," say the authors. "Efforts to decrease mortality in the period after pancreas transplantation should be prioritized."

Rebecca Ireland

Original article Browne, S. et al. The impact of pancreas transplantation on kidney allograft survival. Am. J. Transplant. doi:10.1111/j.1600-6143.2011.03627.x 